\title{
The influence of dietary diversity on the nutritional status of children between 6 and 23 months of age in Tanzania
}

\author{
Ahmed Gharib Khamis ${ }^{{ }^{*}}$ (D), Akwilina Wendelin Mwanri ${ }^{2}$, Julius Edward Ntwenya ${ }^{3}$ and Katharina Kreppel ${ }^{4,5}$
}

\begin{abstract}
Background: Undernutrition poses a serious health challenge in developing countries and Tanzania has the highest undernutrition burden of Eastern and Southern Africa. Poor infant and young child feeding practices have been identified as the main causes for undernutrition. As dietary diversity is a major requirement if children are to get all essential nutrients, it can thus be used as one of the core indicators when assessing feeding practices and nutrition of children. Therefore, adequate information on the association between dietary diversity and undernutrition to identify potential strategies for the prevention of undernutrition is critical. Here we examined to what extent dietary diversity is associated with undernutrition among children of 6 to 23 months in Tanzania.
\end{abstract}

Methods: Using existing data from the Tanzania Demographic and Health Survey of 2015-2016, we carried out secondary data analysis. Stunting, Wasting and Underweight of the surveyed children were calculated from Z-scores of Height-for-age (HAZ), Weight-for-height (WHZ) and Weight-for-age (WAZ) based on 2006 WHO standards. A composite dietary diversity score was created by summing the number of food groups eaten the previous day as reported for each child by the mother ranging from 0 to 7 . Then, minimum dietary diversity (MDD) of 4 food groups out of seven was used to assess the diversity of the diet given to children. Bivariate and multivariate logistic regression techniques were used to assess the crude and adjusted odds ratios of stunting, wasting and being underweight.

Results: A total of 2960 children were enrolled in this study. The prevalence of stunting was 31\%, wasting $6 \%$ and underweight $14 \%$. Among all children, 51\% were female and $49 \%$ male. The majority (74\%) of children did not reach the MDD. The most commonly consumed types of foods were grains, roots and tubers (91\%), and Vitamin A containing fruits and vegetables (65\%). The remaining food groups were reported to be consumed by a much lower proportion of children, including eggs (7\%), meat and fish (36\%), milk and dairy products (22\%), as well as legumes and nuts (35\%), and other vegetables (21\%). Consumption of a diverse diet was significantly associated with a reduction of stunting, wasting and being underweight in children. The likelihood of being stunted, wasted and underweight was found to decrease as the number of food groups consumed increased. Children who did not receive the MDD had a significantly higher likelihood of being stunted ( $A O R=1.37,95 \% \mathrm{Cl} ; 1.13-1.65)$ and underweight $(\mathrm{AOR}=1.49,95 \% \mathrm{Cl}$; 1.15-1.92), but this was not the case for wasting. Consumption of animal-source foods has been found to be associated with reduced stunting among children.

Conclusion: Consumption of a diverse diet is associated with a reduction in undernutrition among children of 6 to 23 months in Tanzania. Measures to improve the type of complementary foods in order to meet the energy and nutritional demands of children should be considered in Tanzania.

Keywords: Dietary diversity, Complementary feeding, Undernutrition, Pediatric, Infants and young children, Tanzania

\footnotetext{
*Correspondence: ahmadboycd@gmail.com

'Department of Epidemiology and Biostatistics, Muhimbili University of

Health and Allied Sciences, Dar-es-Salaam, Tanzania

Full list of author information is available at the end of the article
}

(c) The Author(s). 2019 Open Access This article is distributed under the terms of the Creative Commons Attribution 4.0 International License (http://creativecommons.org/licenses/by/4.0/), which permits unrestricted use, distribution, and reproduction in any medium, provided you give appropriate credit to the original author(s) and the source, provide a link to the Creative Commons license, and indicate if changes were made. The Creative Commons Public Domain Dedication waiver (http://creativecommons.org/publicdomain/zero/1.0/) applies to the data made available in this article, unless otherwise stated. 


\section{Background}

Undernutrition poses a serious challenge in developing countries [1]. The World Health Report of 2016 estimated that about 155 million children under five years of age are stunted and 52 million wasted [2]. The rates of undernutrition were substantially higher in the SubSaharan African (SSA) region [1, 3]. In Tanzania, a high prevalence of chronic and acute undernutrition still persists. It was estimated that about 450,000 children in Tanzania are acutely undernourished or wasted, with over 100,000 suffering from the most severe form of acute malnutrition [4], making it a country with one of the highest undernutrition burdens in Eastern and Southern Africa [5]. The recently released national survey of 2018 reported that $31.8 \%$ of children were stunted, $3.6 \%$ were wasted and $14 \%$ were underweight [6]. The damage caused by undernutrition during the first two years of life may compromise cognitive development of the children and in turn result in poor educational achievement, low economic productivity, and is associated with illness and mortality during adulthood [7].

Poor infant and young child feeding practices are the main causes of undernutrition in Tanzania $[8,9]$ and other developing countries [2]. From the age of 6 months, breastfeeding is no longer able to meet all nutritional requirements of a growing child, and therefore, the consumption of adequate, diversified food is necessary [10, 11]. Globally, however, only less than one-fourth of infants aged 6-23 months meet the recommended criteria for dietary diversity, and only a few of them are receiving a nutritionally adequate diet [2]. The World Health Organization has recommended that an infant should receive the minimum dietary diversity (MDD) of at least four food groups out of seven in order to maintain proper growth and development during this critical period [12], but many children cannot meet these criteria. In Tanzania for example, according to the recent report, only $35.1 \%$ of children aged 6 to 23 months had received the MDD [13], and only $23.3 \%$ in Ethiopia [14]. Dietary diversity, as a marker of micronutrient adequacy, may increase nutrient density of the complementary foods [15], which promote optimal child growth and development. Receiving an inadequately diversified diet may lead to undernutrition [16], and predispose children to opportunistic infections and severe illnesses [7].

Although the association between dietary diversity and the nutritional status of children has already been studied in various countries [16-21], studies that use large-scale data are scarce, particularly in Tanzania. In addition, many previous studies in Tanzania have largely focused on other aspects of child nutrition, like micronutrient content [9], complementary feeding practices $[9,22-25]$ and their determinants [26, 27]. None of them, according to our literature review, has examined the association between child diet diversity and nutritional status. Understanding the influence of dietary diversity on the nutritional status of children could be useful to inform nutrition policy and propose interventions that focus on improving the quality of complementary foods. Findings from this study will therefore be important to public health experts in Tanzania and help to work towards the Sustainable Development Goal-2 (SDG-2) agenda, which aims to end malnutrition in all its forms by 2030 [28]. The present study examined the role of child dietary diversity on undernutrition in Tanzania by using the large available dataset, which is representative of the whole country.

\section{Methods}

\section{Data source}

This is a secondary data analysis from data of the Tanzania Demographic and Health Survey (TDHS) 2015-2016 which was implemented by the National Bureau of Statistics in collaboration with other government partners [13]. Data collection procedures have been described and published in the TDHS 2015 report [13]. Briefly, TDHS of 2015 was designed to produce representative samples at national, regional and ruralurban levels. The TDHS of 2015 was part of the worldwide demographic and health survey program, aimed at assisting countries to collect data to monitor and evaluate their population, health and nutrition programs. The survey employed a two-stage sampling design. In the first-stage, 608 clusters were randomly selected. The second-stage sampling involved systematic sampling of 22 households from the selected clusters. A total of 13, 376 households were included. In this analysis, only data of children aged between 6 to 23 months during the study, matched with their mothers, were finally selected for further analysis.

We used the Kids file (KR file) and Mother file (MR file) from the TDHS data obtained from an online data repository to get information about nutrition status and dietary diversity of children [20]. From 10,233 children of 0-59 month old assessed in the survey, we selected 2960 younger children aged between 6 and 23 months living with their mothers. This was because in the TDHS, information on feeding practices was collected only for the youngest child of this age group. Details of the procedure used to select children for this study are described in Fig. 1.

\section{Nutrition status calculation}

Weight and height were recorded by directly measuring children to generate anthropometric variables used in this study [29]. Stunting, wasting and underweight were calculated from height-for-age z-scores (HAZ), weightfor-height z-scores (WHZ), and weight-for-age z-scores (WAZ); based on 2006 WHO standards [29]. Height-forage z-scores are used to measure chronic malnutrition 


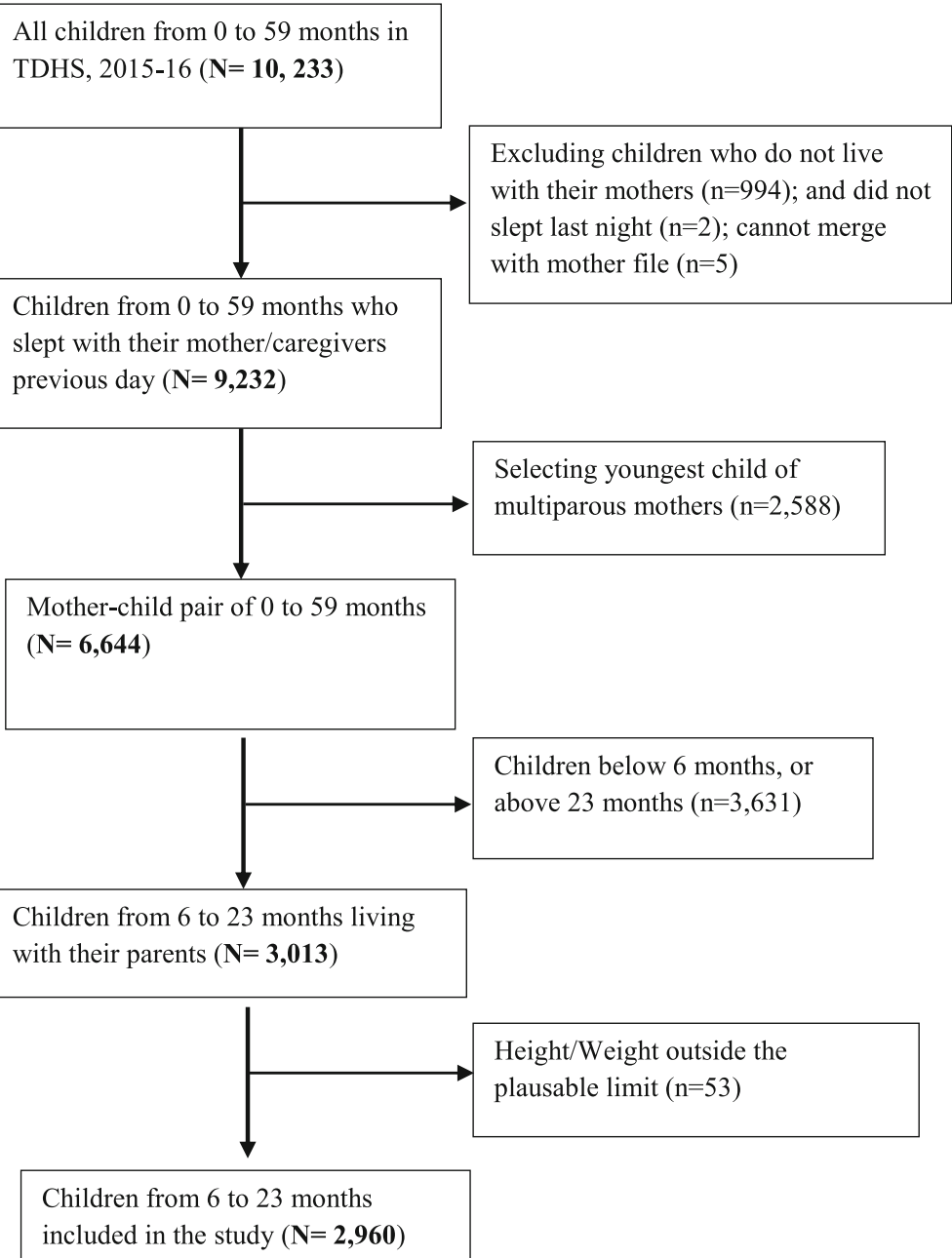

Fig. 1 Selection of children of 6 to 23 months from TDHS 2015-16

due to prolonged food deprivation. Weight-for-height z-scores captures undernutrition due to recent food deprivation and malnutrition, and WAZ measures the child's body mass relative to its chronologic age, and used as a proxy for underweight. Children whose HAZ, WHZ and WAZ was below minus two standard deviations (-2 SD) from the median of the reference population were considered short for their age (stunted), wasted and underweight respectively [29]. We excluded missing values and biologically implausible values in our study as shown in Fig. 1.

\section{Dietary diversity calculation}

To measure dietary diversity, we adopted the WHO's Infant and Young Children Feeding guidelines (IYCF) [12] as an internationally acceptable complementary feeding guideline. The dataset used in the analysis contains information on food items used to calculate the diet diversity indicator. The TDHS survey collected information on food items a child consumed throughout the previous day. We categorized these food items into seven major food groups based on the WHO's IYCF guidelines. These food groups are: (i) grains, roots, and tubers; (ii) legumes and nuts; (iii) flesh foods (meat, fish, poultry and liver/organ meats); (iv) eggs; (v) vitamin A rich fruits and vegetables; (vi) dairy products (milk, yogurt, cheese); (vii) other fruits and vegetables. If a child consumed at least one food item from a food group throughout the previous day, the group was assigned a value of one (1) for that child, and zero (0) if not consumed. The group scores are then summed up to obtain the dietary diversity score, which ranges from zero to seven, whereby zero represents non-consumption of any of the food items in the food groups, and seven represents the highest level of diet diversification. The MDD was attained if a child had consumed four or more food groups $(\mathrm{FG} \geq 4)$ out of the seven food groups over the previous day. 


\section{Other covariates}

Based on the reviewed literature and the objective of this study we considered other characteristics of the child reported by the mother. A child's characteristics included in the study were age, gender, and its size at birth as perceived subjectively by the mother, as well as place of birth and any presence of fever and diarrhea in the past two weeks before the interview $[18,21]$.

\section{Statistical analysis}

We presented categorical variables as proportions, and continuous variables as means and standard deviation (Means $\pm \mathrm{SD})$. To determine the relationship between dietary diversity and nutrition status outcomes, i.e. stunting, wasting and underweight, we first built a series of bivariate logistic regression (for dichotomous outcomes) models. Dietary diversity score, the independent variable of interest, was captured as a continuous variable from the count of the number of food groups the child consumed the previous day before the survey. A separate model was created for each anthropometric outcome, with dietary diversity scores as the independent variable. In addition, the same was done for the MDD as a categorical indicator. Each food group was entered in a bivariate and multivariate model to test its association with nutrition status of the children. The multivariable models for each outcome were controlled for the child's gender, age, presence of fever (yes/no), and diarrhea (yes/no) in the past two weeks before the survey $[18,21]$. Results were considered significant if $p<0.05$. All analyses were performed using Statistical Package for the Social Science (SPSS) software version 23, Stata software, and graphically visualized in Excel.

\section{Results}

\section{Characteristics of the children}

Table 1 presents the general characteristics of the 2960 children enrolled in this study. Among them, 51 and $49 \%$ were female and male respectively. Their mean (SD) age was 14.2 (5.1) months with about 64 and $34 \%$ born at health facility and at home, respectively. Among the studied children, $31 \%$ were stunted, $6 \%$ wasted and $14 \%$ underweight. More than 60\% had normal birth weight of above $2.5 \mathrm{~kg}$. About $79 \%$ of children had no diarrhea within two weeks before the survey, and $78 \%$ had no sign of fever.

\section{Dietary diversity of 6 to 23 month old children}

Fig. 2 shows the proportion of children in terms of food groups consumed in the previous day before the survey. This analysis found that the majority (74\%) of children did not meet the recommended MDD of more than 4 food groups the previous day. Only (26\%) of them had received a diversified diet (Fig. 3). The most commonly
Table 1 Descriptive characteristics of the children aged 6-23 months included in the study $(N=2960)$

\begin{tabular}{ll}
\hline Variable & Mean (SD) and Frequency (\%) \\
\hline Age of Children (months) & $14.2($ SD $=5.1$ months) \\
Prevalence of undernutrition & \\
Stunting (HAZ $<-2 S D)$ & $927(31 \%)$ \\
Wasting (WHZ $<-2 S D)$ & $179(6 \%)$ \\
Underweight (WAZ<-2SD) & $423(14 \%)$ \\
Gender & \\
Male & $1459(49 \%)$ \\
Female & $1501(51 \%)$ \\
Place of Birth & $1913(64 \%)$ \\
Health facility & $1001(34 \%)$ \\
At home & $46(2 \%)$ \\
Other places & \\
Size at Birth & $1801(61 \%)$ \\
Above 2.5 kg & $100(3 \%)$ \\
Below 2.5 kg & $1059(36 \%)$ \\
Not reported & \\
Presence of Diarrhea & $625(21 \%)$ \\
YES & $2335(79 \%)$ \\
NO & \\
Presence of Fever & \\
YES & \\
NO & \\
\hline
\end{tabular}

*HAZ Height-for-Age Z-scores, WHZ Weight-for-Height Z-scores, WAZ Weightfor-Age Z-scores, SD Standard deviation.

consumed types of foods were grains, roots and tubers (91\%) and vitamin A containing fruits and vegetables (65\%). Lesser proportions of children were reported to have consumed food items of the remaining food groups including eggs (7\%), meat and fish (36\%), milk and dairy products $(22 \%)$, as well as legumes and nuts (35\%) and other vegetables $(21 \%)$.

\section{Association between dietary diversity and undernutrition}

Table 2 presents the results of an association between dietary diversity and stunting, wasting and underweight. Based on this analysis using the dietary diversity score and MDD as independent variables, consumption of a diverse diet was significantly associated with a reduction in stunting, wasting and being underweight for children. The likelihood of suffering from stunting, wasting and underweight was found to decrease as the number of food groups consumed increased. Therefore, both the dietary diversity score and MDD analysis showed that children who consumed diverse diets are less likely to be undernourished than those who have a less diverse diet. In an adjusted model, children who did not receive the MDD had a significantly higher likelihood of becoming 


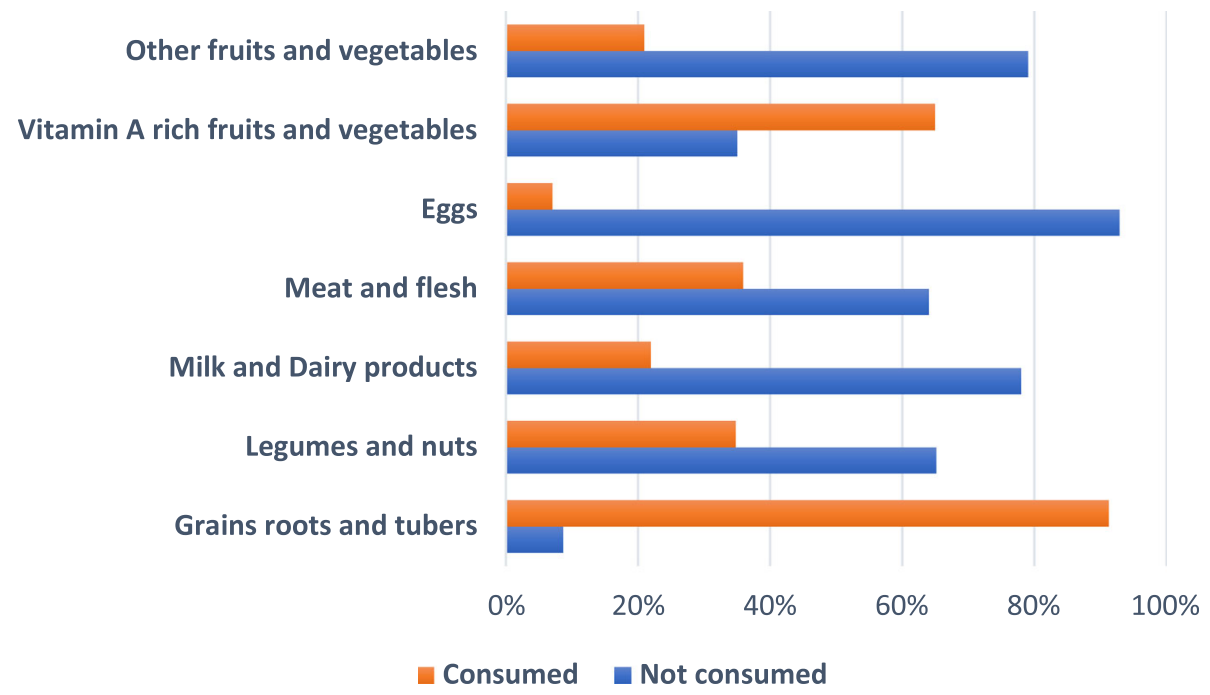

Fig. 2 Proportion of children in terms of their food consumption

stunted $(\mathrm{AOR}=1.37,95 \% \mathrm{CI} ; 1.13-1.65)$ and underweight $(\mathrm{AOR}=1.49,95 \% \mathrm{CI} ; 1.15-1.92)$ compared to children who received the MDD. However; there was no association between the consumption of the MDD and wasting (unadjusted: $\mathrm{OR}=1.19$, 95\% CI 0.83-1.71; adjusted: $\mathrm{AOR}=1.18,95 \% \mathrm{CI} ; 0.82-1.69$ ).

Table 3 shows unadjusted and adjusted odds from bivariate and multivariable logistic regression models for the association between specific food groups and stunting, wasting and underweight. The consumption of milk, meat and eggs has been found to be associated with reduced stunting among children. Children who did not consume any milk (AOR $=1.34 ; 95 \% \mathrm{CI} ; 1.09-1.63$ ), meat $(\mathrm{AOR}=1.27 ; 95 \% \mathrm{CI} ; 1.07-1.53)$, and eggs $(\mathrm{AOR}=$ 1.46; $95 \% \mathrm{CI}$; $1.05-2.03$ ) have a higher likelihood of becoming stunted in the adjusted model. On the other hand, children who did not consume any grains had a higher likelihood of becoming wasted (AOR $=1.62$; 95\% CI; 1.02-2.58). Similarly, children who did not consume any legumes and nuts had a higher likelihood of becoming wasted in the unadjusted model $(\mathrm{OR}=1.42 ; 95 \% \mathrm{CI}$; 1.0-1.96).

Moreover, the analysis shows that children who did not consume any egg or food made from eggs were more likely to be underweight. The likelihood of becoming underweight for children who did not consume any eggs is more than twice as high compared to children who consumed egg products $(\mathrm{AOR}=2.1 ; 95 \% \mathrm{CI} ; 1.29-3.69)$. In addition, the likelihood of becoming underweight for children who did not consume vitamin A rich fruits and vegetables is higher compared to children who do $(\mathrm{AOR}=$ 1.46; 95\% CI; 1.17-1.83).

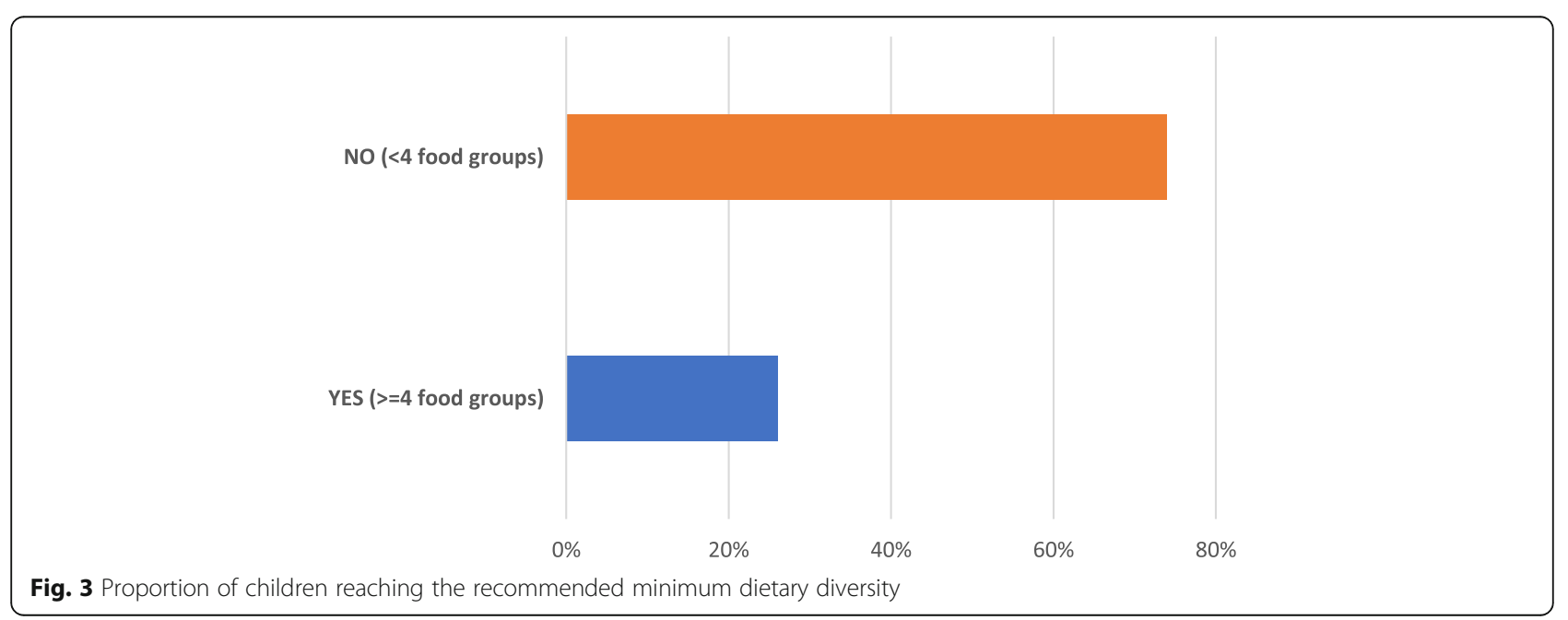


Table 2 Bivariate and multivariate logistic regression analysis of the association between dietary diversity and stunting, wasting and underweight

\begin{tabular}{|c|c|c|c|c|c|c|c|c|c|}
\hline \multirow[b]{2}{*}{ Diversity score } & \multicolumn{3}{|c|}{ Stunting (HAZ<-2SD) } & \multicolumn{3}{|c|}{ Wasting $(\mathrm{WHZ}<-2 \mathrm{SD})$} & \multicolumn{3}{|c|}{ Underweight (WAZ <-2SD) } \\
\hline & $n$ & OR $(95 \% \mathrm{Cl})$ & $\mathrm{AOR}^{\mathrm{a}}(95 \% \mathrm{Cl})$ & $n$ & OR $(95 \% \mathrm{Cl})$ & $\mathrm{AOR}^{\mathrm{a}}(95 \% \mathrm{Cl})$ & $n$ & OR $(95 \% \mathrm{Cl})$ & $\operatorname{AOR}^{a}(95 \% \mathrm{Cl})$ \\
\hline 0 & 18 & Ref. & Ref. & 8 & Ref. & Ref. & 11 & Ref. & Ref. \\
\hline 1 & 93 & $0.8(0.43-1.47)$ & $0.65(0.35-1.23)$ & 29 & $0.56(0.24-1.3)$ & $0.5(0.21-1.18)$ & 54 & $0.7(0.37-1.6)$ & $0.67(0.32-1.39)$ \\
\hline 2 & 285 & $0.94(0.53-1.69)$ & $0.57(0.31-1.05)$ & 50 & $0.34(0.15-0.76)^{* *}$ & $0.3(0.13-0.7)^{* *}$ & 134 & $0.69(0.35-1.38)$ & $0.52(0.26-1.06)$ \\
\hline 3 & 311 & $1.08(0.61-1.94)$ & $0.61(0.33-1.12)$ & 51 & $0.35(0.15-0.78)^{*}$ & $0.32(0.14-0.73)^{* *}$ & 136 & $0.71(0.35-1.41)$ & $0.52(0.26-1.05)$ \\
\hline 4 & 166 & $0.91(0.5-1.64)$ & $0.49(0.26-0.91)^{*}$ & 31 & $0.35(0.15-0.82)^{*}$ & $0.32(0.13-0.76)^{*}$ & 67 & $0.56(0.27-1.14)$ & $0.4(0.19-0.83)^{*}$ \\
\hline 5 & 46 & $0.68(0.35-1.31)$ & $0.36(0.18-0.72)^{* *}$ & 9 & $0.3(0.11-0.82)^{*}$ & $0.26(0.09-0.74)^{*}$ & 19 & $0.46(0.20-1.04)$ & $0.32(0.14-0.74)^{* *}$ \\
\hline 6 & 6 & $0.44(1.55-1.25)$ & $0.25(0.85-0.72)^{*}$ & 1 & $0.17(0.02-1.49)$ & $0.16(0.19-1.38)$ & 1 & $0.12(0.01-0.98)^{*}$ & $0.09(0.01-0.74)^{*}$ \\
\hline 7 & 2 & $0.51(0.9-2.67)$ & $0.26(0.05-1.38)$ & 0 & - & - & 1 & $0.44(0.51-3.88)$ & $0.31(0.03-2.7)$ \\
\hline \multicolumn{2}{|c|}{ Minimum Dietary Diversity } & OR $(95 \% \mathrm{Cl})$ & $\operatorname{AOR}^{a}(95 \% C l)$ & & OR $(95 \% \mathrm{Cl})$ & $\operatorname{AOR}^{\mathrm{a}}(95 \% \mathrm{Cl})$ & & OR $(95 \% \mathrm{Cl})$ & $\operatorname{AOR}^{a}(95 \% C l)$ \\
\hline \multicolumn{2}{|c|}{ YES ( $\geq 4$ food groups) } & Ref. & Ref. & & Ref. & Ref. & & Ref. & Ref. \\
\hline \multicolumn{2}{|c|}{ NO ( $<4$ food groups) } & $1.19(0.99-1.42)$ & $1.37(1.13-1.65)^{* *}$ & & $1.19(0.83-1.71)$ & $1.18(0.82-1.69)$ & & $1.4(1.08-1.79)^{* *}$ & $1.49(1.15-1.92)^{* *}$ \\
\hline
\end{tabular}

${ }^{* *} P<.01,{ }^{*} P<.05 ;{ }^{a}$ Adjusted for age, gender, diarrhea and fever, Cl confidence interval, OR Odds ratio, AOR Adjusted Odds ratio, Ref reference category. Both models fit the data equally well (all $P>0.10$ in likelihood ratio test); HAZ Height-for-Age Z-scores, WHZ Weight-for-Height Z-scores, WAZ Weight-for-Age Z-scores, SD Standard deviation.

Table 3 Bivariate and multivariate logistic regression analysis of the association between food groups and stunting, wasting and underweight

\begin{tabular}{|c|c|c|c|c|c|c|}
\hline \multirow[t]{3}{*}{ Variable } & \multicolumn{2}{|c|}{ Stunting (HAZ<-2SD) } & \multicolumn{2}{|c|}{ Wasting (WHZ <-2SD) } & \multicolumn{2}{|c|}{ Underweight (WAZ <-2SD) } \\
\hline & Crude & Model $^{\mathrm{a}}$ & Crude & Model $^{a}$ & Crude & Model $^{a}$ \\
\hline & OR $(95 \% \mathrm{Cl})$ & AOR (95\% Cl) & OR $(95 \% \mathrm{Cl})$ & AOR (95\% Cl) & OR $(95 \% \mathrm{Cl})$ & AOR (95\% Cl) \\
\hline \multicolumn{7}{|c|}{ FOOD GROUPS } \\
\hline \multicolumn{7}{|c|}{ Grains roots and tubers } \\
\hline YES & Ref. & Ref. & Ref. & Ref. & Ref. & Ref. \\
\hline NO & $0.89(0.67-1.17)$ & $1.05(0.78-1.41)$ & $1.59(1.01-2.52)^{*}$ & $1.62(1.02-2.58)^{*}$ & $1.22(0.86-1.72)$ & $1.34(0.94-1.90)$ \\
\hline \multicolumn{7}{|c|}{ Legumes and nuts } \\
\hline YES & Ref. & Ref. & Ref. & Ref. & Ref. & Ref. \\
\hline NO & $0.9(0.77-1.06)$ & $0.96(0.81-1.14)$ & $1.4(1.0-1.96)^{*}$ & $1.38(0.98-1.94)$ & $1.1(0.87-1.35)$ & $1.1(0.89-1.39)$ \\
\hline \multicolumn{7}{|c|}{ Milk and Dairy products } \\
\hline YES & Ref. & Ref. & Ref. & Ref. & Ref. & Ref. \\
\hline NO & $1.38(1.13-1.67)^{* *}$ & $1.34(1.09-1.63)^{* *}$ & $1.26(0.85-1.86)$ & $1.24(0.83-1.83)$ & $1.26(0.97-1.64)$ & $1.22(0.94-1.59)$ \\
\hline \multicolumn{7}{|c|}{ Meat and flesh } \\
\hline YES & Ref. & Ref. & Ref. & Ref. & Ref. & Ref. \\
\hline NO & $1.07(0.91-1.26)$ & $1.27(1.07-1.53)^{* *}$ & $1.0(0.73-1.38)$ & $0.97(0.7-1.34)$ & $0.98(0.79-1.22)$ & $1.04(0.89-1.3)$ \\
\hline \multicolumn{7}{|l|}{ Eggs } \\
\hline YES & Ref. & Ref. & Ref. & Ref. & Ref. & Ref. \\
\hline NO & $1.37(0.99-1.89)$ & $1.46(1.05-2.03)^{*}$ & $1.92(0.89-4.15)$ & $1.95(0.9-4.23)$ & $2.1(1.24-3.52)^{* *}$ & $2.1(1.29-3.69)^{* *}$ \\
\hline \multicolumn{7}{|c|}{ Vitamin A rich fruits and vegetables } \\
\hline YES & Ref. & Ref. & Ref. & Ref. & Ref. & Ref. \\
\hline NO & $0.93(0.79-1.1)$ & $1.18(0.99-1.41)$ & $1.29(0.95-1.76)$ & $1.29(0.94-1.78)$ & $1.29(1.04-1.59)^{*}$ & $1.46(1.17-1.83)^{* *}$ \\
\hline \multicolumn{7}{|c|}{ Other fruits and vegetables } \\
\hline YES & Ref. & Ref. & Ref. & Ref. & Ref. & Ref. \\
\hline NO & $1.01(0.83-1.22)$ & $1.07(0.88-1.31)$ & $0.88(0.61-1.26)$ & $0.9(0.63-1.29)$ & $1.06(0.82-1.37)$ & $1.11(0.86-1.44)$ \\
\hline
\end{tabular}

${ }^{* *} P<.01,{ }^{*} P<.05 ;{ }^{a}$ Adjusted for age, gender, diarrhea and fever; $C l$ confidence interval, OR Odds ratio, AOR Adjusted Odds ratio, Ref reference category, HAZ Height-for-Age Z-scores, WHZ Weight-for-Height Z-scores, WAZ Weight-for-Age Z-scores, SD Standard deviation 


\section{Discussion}

This study aimed to examine the association between dietary diversity and undernutrition of children aged 6 to 23 months in Tanzania. In this study, dietary diversity was found to be a protective factor against stunting (HAZ) and underweight (WAZ) among children in Tanzania. These results are consistent with findings reported from other developing countries like Burkina Faso [19], Bangladesh [30], Ethiopia [18], and others [16, 21]. Our study suggests that undernutrition can be reduced by improving the dietary diversity of complementary foods in Tanzania. This is supported by the fact that dietary diversity is a good predictor of dietary quality and micronutrient density in children [15, 31]. Therefore, generally, this makes dietary diversity one of the important factors that policy makers should adopt to improve the nutritional status of children in the country.

However, this study did not find an association between wasting and the dietary diversity of children using the MDD indicator. This is in line with findings from other studies [19, 32]. As a previous study from Ethiopia has shown, wasting is more likely to be associated with diseases or household food insecurity rather than dietary diversity [18]. This might be due to the fact that wasting refers to acute malnutrition as a result of shorter-term episodes of inadequate feeding or illnesses [33].

Our study shows that only a small proportion (26\%) of children of 6 to 23 months old in Tanzania reached the MDD. This is identical to a finding by Ochieng et al. who looked at factors affecting household nutrition security in Tanzania, which reported that only $26 \%$ of children had reached the MDD [27]. Overall, the present study shows that consumption of animal-source foods like meat, milk and eggs is not very common among children of 6-23 months in Tanzania. Similarly, the study by Ochieng et al. reported that meat and fish were consumed by less than $10 \%$ of children under five years [27]. These outcomes are not surprising considering the seasonal and geographical unavailability of some foods, the restrictions imposed by traditions, and possible financial constraints in Tanzania [34]. In this study, children who did not consume any meat were more likely to become stunted. This result corresponds with a survey of 12 to 59 month old children in Cambodia, which concluded that the consumption of animal-source foods was a protective factor against stunting and underweight [17]. Animal-source foods like meat, milk, eggs and poultry have a variety of micronutrients including vitamin A, vitamin B-12, riboflavin, calcium, iron and zinc that are difficult to obtain in adequate quantities from plant sourced foods alone [35]. Thus, insufficient intake of these nutrients may hinder the physical development of a child, resulting in stunting [2]. We therefore highly recommend that public health officials should educate parents and caregivers on the importance of animal-source foods for their children.

Moreover, our analysis emphasizes that the consumption of milk and dairy products is very beneficial for growing children. We found that non-consumption of milk products is a predictor of stunting among children. Failure to give any milk or dairy product at this critical age may result in protein imbalance [2]. Milk is one of the basic foods which can easily provide adequate nutrition when consumed regularly in relative small quantities and which can promote a child's health and is available throughout the year [36]. Therefore, health education messages related to the importance of milk and dairy products as complementary foods is a critical public-health intervention in Tanzania.

Another interesting finding in this study was the association of vitamin A fruits and vegetables with underweight among children. In this study, children who did not consume vitamin A containing fruits and vegetables were more likely to become underweight. Similar findings were reported from a study among children in Ghana [21]. Also, previous research has shown a significant association between Vitamin A intake and undernutrition, but not among infants [37]. Vitamin A is known as an essential micronutrient for growth and immunity. Its deficiency is one of the most important causes of preventable childhood blindness and is a major contributor to morbidity and mortality from infections [37]. Therefore, based on these findings, lack of adequate vitamin A intake may results in the increase of underweight children in the country. It is therefore important for mothers to increase the inclusion of foods rich in vitamin A such as spinach, mangoes and papaya as complementary foods given to their children. These foods are relatively cheap in Tanzania and are easily accessible in both rural and urban areas for most of the year.

We also found that, children who did not consume any food made from grains or legumes were more likely to become wasted. In many countries, including Tanzania, grains and cereals including maize, sorghum, millet and rice are among the first foods that are introduced at the beginning of the complementary feeding period to infants $[9,22,23,38]$. These are very beneficial for the children because they are an excellent source of energy, vitamins and minerals [38]. Therefore, to reduce the prevalence of undernutrition, mothers should provide adequate foods that provide adequate energy and all nutrients to their children.

However, it is important to mention some important limitation of this study. We only considered the dietary diversity as indicator of the overall quality of the child's diet. This study did not take into account the quantity of the foods consumed, and is therefore not able to reliably 
predict that foods consumed met the required dietary intake. Also, due to the cross-sectional nature of this study, a cause and effect relationship cannot be assured and we have to interpret our findings with caution. Additionally, some indicators of the nutrition status like stunting represent a long-term cumulative process, whereas the dietary information available in the TDHS reflects only the previous day before the survey. In addition, the responses given by mothers/caregivers are sometime based on their ability to recall types of foods and their desire to please the surveyor. In spite of the mentioned limitations however, this present study can shed light on how dietary diversity is likely to influence the development of undernutrition in Tanzania. Future, large scale studies are needed to identify the causal relationship between diet and the physical and mental condition of the children in Tanzania.

\section{Conclusion}

This study shows that consumption of a diverse diet is associated with a reduction in undernutrition among children of 6 to 23 months of age in Tanzania. In addition to dietary diversity, animal-source foods like meat, milk and eggs can prevent stunting in children. Especially the consumption of milk products is very important in order to reduce stunting among children. Measures to improve the type of complementary foods given to children to meet their needs for energy and nutrients should be considered. Moreover, strong commitment by the government and public health officials is needed to ensure adequate foods and education on what to feed are available.

\section{Abbreviations \\ AOR: Adjusted Odd Ratio; Cl: Confidence Intervals; FG: Food Group; HAZ: Height-for-Age Z-scores; IYCF: Infant and Young Child Feeding; MDD: Minimum Dietary Diversity; OR: Odd Ratio: SD: Standard Deviation; TDHS: Tanzania Demographic and Health Survey; WAZ: Weight-for-Age Z- scores; WHO: World Health Organisation; WHZ: Weight-for-Height Z-scores}

\section{Acknowledgements}

We acknowledge the support in terms of research trainings received from the consortium Afrique One African Science Partnership for Intervention Research Excellence [Afrique One-ASPIRE /DEL-15-008].

\section{Author's contributions}

AGK conceived the study, analyzed the data and wrote the first draft of the manuscript. AWM, JEN and KK reviewed and revised the manuscript. All authors have read and approved the final version of the manuscript.

\section{Funding}

The data is freely available and no funding was required.

\section{Availability of data and materials}

Data for this study are freely available upon request from the Demographic and health survey (DHS) portal (www.dhsprogram.com).

\section{Ethics approval and consent to participate}

The permission to do this study was given by demographic and health surveys (DHS) program. Since we only used secondary, anonymized data, personal consent was not required.
Consent for publication

Not applicable for this study.

\section{Competing interests}

The authors declare that they have no competing interests.

\section{Author details}

${ }^{1}$ Department of Epidemiology and Biostatistics, Muhimbili University of Health and Allied Sciences, Dar-es-Salaam, Tanzania. ${ }^{2}$ Department of Food Technology, Nutrition and Consumer Sciences, Sokoine University of Agriculture, P. O Box 3006, Chuo Kikuu, Morogoro, Tanzania. ${ }^{3}$ Department of Public Health, The University of Dodoma, P.O. Box 395, Dodoma, Tanzania. ${ }^{4}$ School of Life Sciences and Bio-Engineering, Nelson Mandela African Institution of Science and Technology, Arusha, Tanzania. ${ }^{5}$ Department of Environmental Health and Ecological Sciences, Ifakara Health Institute, Dar-es-Salaam, Tanzania.

Received: 14 October 2019 Accepted: 22 December 2019

Published online: 28 December 2019

\section{References}

1. Van de Poel E, Hosseinpoor AR, Speybroeck N, Van Ourti T, Vega J. Socioeconomic inequality in malnutrition in developing countries. Bull World Health Organ. 2008:86(4):282-91.

2. WHO. Infant and young child feeding. 2018. Available through https://www. who.int/news-room/fact-sheets/detail/infant-and-young-child-feeding. Retrieved on 21 February 2019

3. UNICEF WHO, Bank W. Joint child malnutrition estimates. Levels and trends in child malnutrition: Key findings of the 2015 edition. Global Database on Child Growth and Malnutrition. New York: UNICEF, WHO, \& World Bank Group; 2015.

4. IRIS: Overcoming the challenges of undernutrition in tanzania through 2021 p. 75-83. Available through https://www.iris-france.org/wp-content/ uploads/2017/03/Tanzania-Final-report.pdf. Retrieved on 10 January 2019.

5. IFPRI: Global Nutrition Report 2014: Actions and Accountability to Accelerate the World's Progress on Nutrition: International Food Policy Research Institute, Washington, DC. Available from http://ebrary.ffpri.org/cdm/ref/ collection/p15738coll2/id/128484. Retrieved on 10 January 2019

6. Ministry of Health, Community Development, Gender, Elderly and Children (MoHCDGEC) [Tanzania Mainland], Ministry of Health (MoH) [Zanzibar], Tanzania Food and Nutrition Centre (TFNC), National Bureau of Statistics (NBS), Office of the Chief Government Statistician (OCGS) [Zanzibar], UNICEF. Tanzania National Nutrition Survey using SMART methodology (TNNS) 2018. Dar es Salaam, Tanzania: MoHCDGEC, MoH, TFNC, NBS, OCGS, and UNICEF; 2018

7. Caulfield LE, de Onis M, Blossner M, Black RE. Undernutrition as an underlying cause of child deaths associated with diarrhea, pneumonia, malaria, and measles. Am J Clin Nutr. 2004;80(1):193-8.

8. Mamiro P, Kolsteren P, Roberfroid D, Tatala S, Opsomer A, Camp J. Feeding practices and factors contributing to wasting, stunting, and iron deficiency anaemia among 3-23-month children in Kilosa District, rural Tanzania. J Health Popul Nutr. 2005;23.

9. Kulwa KBM, Mamiro PS, Kimanya ME, Mziray R, Kolsteren PW. Feeding practices and nutrient content of complementary meals in rural Central Tanzania: implications for dietary adequacy and nutritional status. BMC Pediatr. 2015;15(1):171

10. Dewey K. Guiding principles for complementary feeding of the breastfed child. Washington, DC: Pan American Health Organization/World Health Organization; 2003.

11. Abeshu MA, Lelisa A, Geleta B. Complementary feeding: review of recommendations, feeding practices, and adequacy of homemade complementary food preparations in developing countries - lessons from Ethiopia. Front Nutr. 2016;3:41.

12. WHO: Indicators for assessing infant and young child feeding practices: Part 1 Definitions. Conclusions of a consensus meeting held 6-8 November 2007 in Washington D.C. USA: GenevaWorld Health Organization; 2008.

13. Ministry of Health, Community Development, Gender, Elderly and Children (MoHCDGEC) [Tanzania Mainland], Ministry of Health $(\mathrm{MoH})$ [Zanzibar], National Bureau of Statistics (NBS), Office of the Chief Government Statistician (OCGS), ICF. Tanzania demographic and health survey and malaria Indicator survey (TDHS-MIS) 2015-16. Dar-es-Salaam, Tanzania: Rockville, Maryland, USA: MoHCDGEC, MoH, NBS, OCGS, and ICF; 2016. 
14. Temesgen H, Negesse A, Woyraw W, Mekonnen N. Dietary diversity feeding practice and its associated factors among children age 6-23 months in Ethiopia from 2011 up to 2018: a systematic review and meta-analysis. Ital J Pediatr. 2018:44(1):109.

15. Moursi MM, Arimond M, Dewey KG, Treche S, Ruel MT, Delpeuch F. Dietary diversity is a good predictor of the micronutrient density of the diet of 6-to 23-month-old children in Madagascar. J Nutr. 2008;138(12):2448-53.

16. Arimond M, Ruel MT. Dietary diversity is associated with child nutritional status: evidence from 11 demographic and health surveys. J Nutr. 2004; 134(10):2579-85.

17. Darapheak C, Takano T, Kizuki M, Nakamura K, Seino K. Consumption of animal source foods and dietary diversity reduce stunting in children in Cambodia. Int Arch Med. 2013;6(1):29.

18. Motbainor A, Worku A, Kumie A. Stunting is associated with food diversity while wasting with food insecurity among underfive children in east and west Gojjam zones of Amhara region Ethiopia. PloS One. 2015;10(8):e0133542.

19. Sié A, Tapsoba C, Dah C, Ouermi L, Zabre P, Bärnighausen T, Arzika AM, Lebas E, Snyder BM, Moe C. Dietary diversity and nutritional status among children in rural Burkina Faso. Int Health. 2018;10(3):157-62.

20. Ahmad I, Khalique N, Khalil S. Dietary diversity and stunting among infants and young children: a cross-sectional study in Aligarh. Indian J Commun Med. 2018;43(1):34

21. Frempong RB, Annim SK. Dietary diversity and child malnutrition in Ghana. Heliyon. 2017;3(5):e00298.

22. Kinabo JL, Mwanri AW, Mamiro PS, Kulwa K, Bundala NH, Picado J, Msuya J, Ntwenya J, Nombo A, Mzimbiri R. Infant and young child feeding practices on Unguja Island in Zanzibar, Tanzania: a ProPAN based analysis. Tanzan J Health Res. 2017;19(3).

23. Vitta BS, Benjamin M, Pries AM, Champeny M, Zehner E, Huffman SL. Infant and young child feeding practices among children under 2 years of age and maternal exposure to infant and young child feeding messages and promotions in Dar es Salaam, Tanzania. Matern Child Nutr. 2016;12(Suppl 2): 77-90.

24. Ogbo FA, Ogeleka P, Awosemo AO. Trends and determinants of complementary feeding practices in Tanzania, 2004-2016. Trop Med Health. 2018;46(1):46-0.

25. Nyaruhucha CN, Msuya JM, Mamiro PS, Kerengi AJ. Nutritional status and feeding practices of under-five children in Simanjiro District, Tanzania. Tanzan Health Res Bull. 2006;8(3):162-7.

26. Victor R, Baines S, Agho K, Dibley M. Factors associated with inappropriate complementary feeding practices among children aged 6-23 months in Tanzania. Matern Child Nutr. 2014;10(4):545-61.

27. Ochieng J, Afari-Sefa V, Lukumay PJ, Dubois T. Determinants of dietary diversity and the potential role of men in improving household nutrition in Tanzania. PLoS One. 2017;12(12):e0189022.

28. UN: Transforming our world : the 2030 Agenda for Sustainable Development, 21 October 2015, A/RES/70/1. Retrieved from https://www. refworld.org/docid/57b6e3e44.html . Accessed 10 Apr 2019.

29. WHO: Physical status and the use and interpretation of anthropometry. Reports of WHO expert committee. Technical Report Series: No. 854 Switzerland: Geneva; 2006. p. 13-125.

30. Rah JH, Akhter N, Semba RD, De Pee S, Bloem MW, Campbell A, MoenchPfanner R, Sun K, Badham J, Kraemer K. Low dietary diversity is a predictor of child stunting in rural Bangladesh. Eur J Clin Nutr. 2010;64(12):1393.

31. Bandoh DA, Kenu E. Dietary diversity and nutritional adequacy of underfives in a fishing community in the central region of Ghana. BMC Nutr. 2017;3(1):2.

32. Jones AD, Ickes SB, Smith LE, Mbuya MN, Chasekwa B, Heidkamp RA, Menon P, Zongrone AA, Stoltzfus RJ. World Health Organization infant and young child feeding indicators and their associations with child anthropometry: a synthesis of recent findings. Matern Child Nutr. 2014;10(1): $1-17$.

33. Collins S, Dent N, Binns P, Bahwere P, Sadler K, Hallam A. Management of severe acute malnutrition in children. Lancet. 2006;368(9551):1992-2000.

34. Powell B, Bezner Kerr R, Young SL, Johns T. The determinants of dietary diversity and nutrition: ethnonutrition knowledge of local people in the east Usambara Mountains, Tanzania. J Ethnobiol Ethnomed. 2017;13(1):23.

35. Neumann C, Harris DM, Rogers LM. Contribution of animal source foods in improving diet quality and function in children in the developing world Nutr Res. 2002;22(1-2):193-220.
36. Lanou AJ, Berkow SE, Barnard ND. Calcium, dairy products, and bone health in children and young adults: a reevaluation of the evidence. Pediatr. 2005; 115(3):736-43.

37. Abedi AJ, Mehnaz S, Ansari MA, Srivastava JP, Srivastava KP. Intake of vitamin a \& its association with nutrition status of pre-school children. Int J Commun Med Public Health. 2017;2(4):489-93.

38. Belew AK, Ali BM, Abebe Z, Dachew BA. Dietary diversity and meal frequency among infant and young children: a community based study. Ital J Pediatr. 2017:43(1):73.

\section{Publisher's Note}

Springer Nature remains neutral with regard to jurisdictional claims in published maps and institutional affiliations.
Ready to submit your research? Choose BMC and benefit from:

- fast, convenient online submission

- thorough peer review by experienced researchers in your field

- rapid publication on acceptance

- support for research data, including large and complex data types

- gold Open Access which fosters wider collaboration and increased citations

- maximum visibility for your research: over $100 \mathrm{M}$ website views per year

At BMC, research is always in progress.

Learn more biomedcentral.com/submissions 